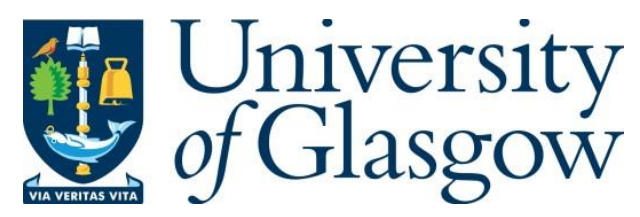

Chen, R. et al. (2018) Variability study of MWCNT local interconnects considering defects and contact resistances - Part I: pristine MWCNT. IEEE Transactions on Electron Devices, 65(11), pp. 4955-4962.

There may be differences between this version and the published version. You are advised to consult the publisher's version if you wish to cite from it.

http://eprints.gla.ac.uk/167764/

Deposited on: 28 August 2018

Enlighten - Research publications by members of the University of Glasgow http://eprints.gla.ac.uk 


\title{
Variability Study of MWCNT Local Interconnects Considering Defects and Contact Resistances - Part I: Pristine MWCNT
}

\author{
Rongmei Chen, Jie Liang, Jaehyun Lee, Vihar P. Georgiev, Raphael Ramos, Hanako Okuno, Dipankar Kalita, \\ Yuanqing Cheng, Liuyang Zhang, Reetu R. Pandey, Salvatore Amoroso, Campbell Millar, Asen Asenov, \\ Jean Dijon, Aida Todri-Sanial
}

\begin{abstract}
In this paper, an enhanced compact model of multiwalled carbon nanotube (MWCNT) interconnects while considering defects and contact resistance is proposed. Based on the atomistic-level simulations, we have found that defect densities impact MWCNT resistance and ultimately their electrical performance. Furthermore, we have computed by atomisticlevel simulations the end-contact resistance between single-wall carbon nanotube (SWCNT) and Palladium (Pd) electrode to mimic the Pd-CNT end-contact resistance of each CNT shell in MWCNT. We have developed an advanced shell-by-shell model to include various parameters such as shell diameter, shell chirality, defects on each shell, and connectivity of each shell to endcontacts. We run Monte Carlo simulations to perform variability studies on each of these parameters to understand the electrical performance variation on MWCNT interconnects. We present simulation results to convey the critical impact of variations. The impact of doping on MWCNT variability in the form of Fermi level shift will be addressed in Part II of this paper.
\end{abstract}

Index Terms-multi-walled carbon nanotubes, compact model, defects, contact resistance, variability, Monte Carlo simulation.

\section{INTRODUCTION}

A S technology scales down, the transistor performance continues to improve. However, for interconnect, scaling leads to higher current density and more substantial resistance. The transition of interconnect material from Aluminum (Al) to Copper $(\mathrm{Cu})$ a few decades ago enabled further scaling of interconnects. Nevertheless, $\mathrm{Cu}$ interconnects resistance rises rapidly as its dimensions scale and become comparable to the order of electron mean free path $(\sim 40 \mathrm{~nm}$ at room temperature) [1], [2]. Compared with $\mathrm{Cu}$, carbon nanotube (CNT) has attractive properties such as ballistic transport, high thermal conductivity, and ampacity [3], [4]. Because of strong $\mathrm{sp}^{2}$

This work was supported by the European Commission H2020 CONNECT project under grant agreement number 688612. (http://www.connecth2020.eu/).

R. Chen, J. Liang, R. R. Pandey and A. Todri-Sanial are with the Microelectronics Department, LIRMM, University of Montpellier, CNRS, Montpellier, 34095 France. E-mails: rongmei.chen@lirmm.fr; aida.todri@lirmm.fr.

R. Ramos and J. Dijon are with the University Grenoble Alpes/CEALITEN, Grenoble, France.

J. Lee, V. P. Georgiev, and A. Asenov are with the School of Engineering, University of Glasgow, Glasgow, G12 8QQ UK.

H. Okuno and D. Kalita are with the University Grenoble Alpes/CEAINAC, Grenoble, France.

S. Amoroso and C. Millar are with Synopsys, Ltd., Glasgow, UK

Y. Cheng and L. Zhang are with the School of Electronic and Information Engineering, Beihang University, Beijing, China, 100191.

Manuscript submitted May 2018. bonding between carbon atoms, CNT is much more resistant to electromigration (EM) and can sustain significantly larger current densities than $\mathrm{Cu}$ [5]. Furthermore, self-heating problem is a concern on deeply scaled $\mathrm{Cu}$ interconnects whereas CNTs have long ballistic electronic transport length $\sim 1 \mu \mathrm{m}$, making them have higher electrical and thermal conductivities and thus capable of carrying high current density with virtually no heating thanks to its 1D electronic structure [6]-[8].

Compared with $\mathrm{Cu}$ line process, the fabrication of CNT requires different processing steps and much higher temperature (up to $900{ }^{\circ} \mathrm{C}$ [9]) to achieve good quality CNT growth. However, achieving BEOL process compatible with CNTs would require to grow them at a lower temperature such as 450 ${ }^{\circ} \mathrm{C}$ to $500{ }^{\circ} \mathrm{C}$. Growing CNTs at such temperatures introduces several variations, i.e., diameter, chirality. Furthermore, at such temperatures defects are omnipresent, and the most typical types are vacancies, interstitials, non-hexagonal rings and grain boundaries [10], [11]. Defects can trap or scatter carriers and thus ultimately impact CNT interconnect performance.

Good contacts between the CNTs and metal electrodes are very challenging. Poor contacts induce a significant contact resistance or might be even disconnected as in the case of inner shells on MWCNTs [12], [13]. This can be a significant concern for vertical interconnects where the electrode acts as a support for the catalytic growth of CNT and also as an electrical contact [14]. Currently, there are two types of contacts with CNTs - side-contacts and end-contacts [15]. The side-contact has the metal deposited on top of the CNT, enabling the electrons to flow along the length of the metalCNT tube interface. End-contacts are formed between the end of CNT tube and metal. The contact resistance depends on the overlap length between the metal and CNT tube. The interface between electrode and CNT for the end-contact is covalent metal-carbon bonds while it is Van der Waals bond for the side contact as discussed and described in [15]. As a result, the end-contact tends to have smaller contact resistance than the side-contact. Whereas, end-contacts in MWCNT may suffer from disconnected shells to the electrodes which ultimately also increases the contact resistance.

In this work, we perform a thorough study on process variations and their impact on MWCNT interconnect performance. We investigate the CNT quantum transport property and use the number of conducting channels $N_{C}$ as a figure of merit for computing the change in CNT conductance due to process 


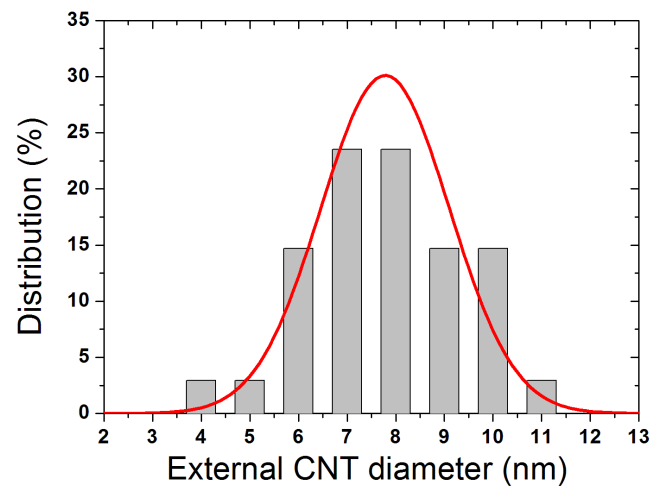

Fig. 1. Experimental results of MWCNT $D_{C N T \max }$ variation. Mean and standard deviation of $D_{C N T \max }$ are $7.8 \mathrm{~nm}$ and $1.6 \mathrm{~nm}$, respectively.

variations. On SWCNTs (with the diameter less than $10 \mathrm{~nm}$ ) the variations in diameter have been shown to have little impact on its conductance [16]. However, on MWCNTs, the diameter variations may change the total number of shells and $N_{C}$; thus, the MWCNT conductance can vary significantly. Chirality determines the property of the CNT, which can be either metallic or semiconducting. Metallic and semiconducting CNT conductivities are significantly different, especially for small diameter CNT [17]. Shell connection to the end-contact also impacts overall MWCNT resistance, and even more so for MWCNT with many shells [18].

To understand how CNT interconnect performance is impacted by these variations, we develop a compact shell-level model capable of combining the MWCNT physical properties with process variations. Such a model is essential for circuitlevel simulation and circuit design space exploration with MWCNT interconnects. The contributions of our work can be summarized as follows.

1) We present a compact shell-level model of MWCNT to include the defects, chirality, end-contact resistance and shell connectivity to end-contacts.

2) We perform Monte Carlo (MC) or best/worst cases simulations to study variations in diameter, chirality, the presence of defects (defect density) and contacts.

\section{SOURCES OF VARIABILITY ON MWCNT}

In this paper, four sources of variations are considered. They are based on observations either from experimental measurements or atomistic-level simulations.

\section{A. Diameter Variations}

For MWCNT, both CNT diameter and number of shells can fluctuate during the fabrication process. Typically, the diameter variation of fabricated CNT shows a Gaussian or normal $(N)$ distribution [19], [20].

By the catalytic CVD method, we grew MWCNT forests which typically exhibit Gaussian distributed outermost diameters $\left(D_{C N T \text { max }}\right)$ with relative variations of $\sim 20 \%$ (standard deviation $=20 \%$ of the mean $D_{C N T \max }$ ), as shown in Fig. 1. However, preliminary experimental results suggest that if

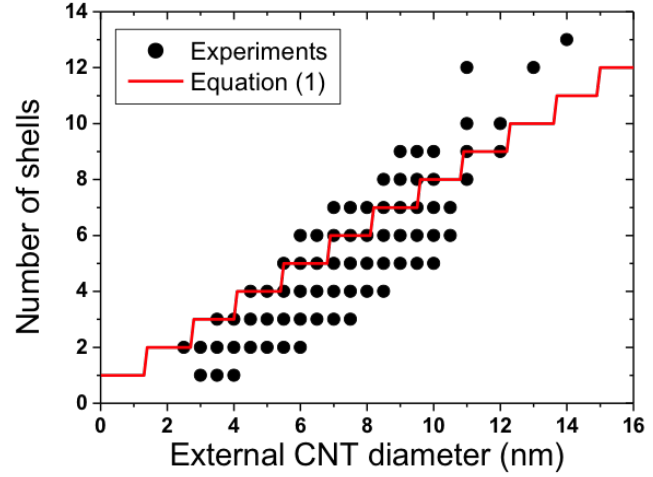

Fig. 2. Experimental results of numbers of shells for MWCNTs of different outermost (external) diameters. Also shown are the calculation results of Equation (1).

each catalyst nanoparticle is confined in a nanoscale via hole [19], the relative diameter variation of individually grown MWCNT can be reduced below $10 \%$. It is believed that this variation, which includes both catalyst size variation and intrinsic CNT growth variation, can be further reduced by improving the nanoscale via hole fabrication process. In this work, to trade off the current achieved diameter variation data and possibly optimized results, we make the assumption that the $D_{C N T \max }$ variation on MWCNTs is a Gaussian distribution with the $\sigma_{D_{C N T \max }}=15 \%$ of the $\mu_{D_{C N T \max }}$, namely $N\left(\mu_{D_{C N \text { T max }}},\left(15 \% \times \mu_{D_{C N \text { T max }}}\right)^{2}\right)$. The $15 \%$ relative variation is an average of the current obtained $20 \%$ and the potentially optimized $10 \%$ relative variation aforementioned. Furthermore, the total number of CNT shells is $p$ and each shell diameter is $D_{C N T i}$, which are calculated by assuming that the shell-to-shell distance is the van der Waals gap $d=0.34 \mathrm{~nm}$ and $D_{C N T \min } / D_{C N T \max }=1 / 2$, in which $D_{C N T \min }$ and $D_{C N T \max }$ are the innermost and outermost CNT shell diameters respectively [16], as shown in Equations (1) and (2) below where "Floor[]" means only the integer part is taken into account.

$$
\begin{gathered}
p=1+\text { Floor }\left[\frac{\left(D_{C N T \max }-D_{C N T \min }\right)}{2 d}\right] \\
D_{C N T i}=D_{C N T \max }-2 d(i-1), \quad 1 \leq i \leq p
\end{gathered}
$$

To support the analytical formulation, we report on highresolution TEM analysis of CNTs grown by hot filament assisted CVD from the alumina-supported iron catalyst. 38 growth experiments were realized under various conditions (catalyst thickness, sample temperature, pressure, hot filament temperature) to grow CNTs with different structures. A systematic TEM analysis was performed, and the diameter and number of shells of about 60 CNTs were measured for each growth condition (i.e., 2300 different CNTs were measured in total). Experimental results on numbers of shells for MWCNT and calculation results based on Equation (1) are presented in Fig. 2. It should be highlighted that Fig. 2 does not represent the variability of CNT structures for a given growth process but 


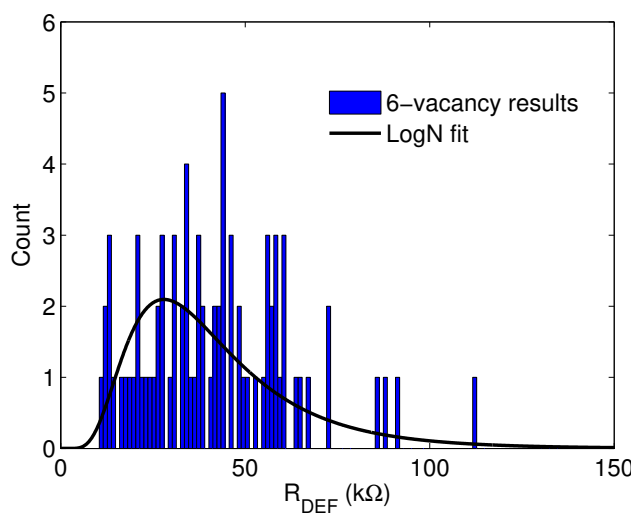

Fig. 3. Atomistic-level simulation results of defect-induced resistance of a SWCNT $(24,0)$ with 6 vacancies. The SWCNT length is set to be about $42 \mathrm{~nm}$ corresponding to a vacancy density of $0.143 / \mathrm{nm}$. Also shown is the corresponding $\log \mathrm{N}$ fit result.

instead illustrates the overall trend observed for hot filament CVD-grown MWCNTs. A similar trend was also reported for thermal CVD grown MWCNTs [21]. From Fig. 2, we can tell that Equation (1) is accurate when $D_{C N T \max }$ is around 11 $\mathrm{nm}$ and the equation over-estimates and under-estimates the number of shells before and after $\sim 11 \mathrm{~nm} D_{C N T \max }$.

\section{B. Defect Occurrence}

Experimental results show that defects in CNT could severely degrade CNT electrical properties through conductivity reduction [22]. In this work, we use vacancy defect as a representative of all defects by assuming that other types of defects may have a similar impact on CNT resistance. Vacancy defect density will be varied to assess the effect of defect density on MWCNTs.

Atomistic-level simulations [23] show that vacancies in a SWCNT induce resistance fluctuation behaving like an Ohmiclike principle, which is proportional to the defect density. Furthermore, due to the statistical distribution of vacancies inside the SWCNT, the resistance induced by the defects $R_{D e f}$ can be fit by a Lognormal $(\log N)$ distribution with mean and standard deviation $10.5117 \Omega$ and $0.5226 \Omega$, respectively (i.e. $\left.\ln \left(R_{D e f}\right) \sim N\left(10.5117,0.5226^{2}\right)\right)$ as shown in Fig. 3. In this paper, we assume that defect-induced resistance variation complies with a $\log N$ distribution with the ratio of $\log N$ parameters mean and standard deviation being 0.5226/10.5117 $=0.0497$. For different defect densities, the average of $R_{D e f}$ (Equation (5)) can be used to calculate $\log N$ mean value of $R_{D e f}$ [24]. In this way, the $\operatorname{LogN}$ distribution of $R_{D e f}$ can be obtained under different defect densities.

\section{CNT Chirality}

Without any particular separation technique, in theory, the probability of a fabricated CNT to be metallic (semiconducting) is $1 / 3(2 / 3)$ [25]. For a MWCNT, the chirality of each shell is also statistically distributed. CNT chirality plays an essential role on CNT parasitics and ultimately its performance [26]. Up to date, there are no experimental data available for distribution of chirality of shells in MWCNT even though chirality can statistically fluctuate during the CNT growth process. In this work, the chirality of each shell in a MWCNT is assumed to be independent and comply with a Bernoulli distribution (or 0-1 distribution) with each shell of $1 / 3$ (or $2 / 3$ ) probability to be metallic (or semiconducting).

\section{Shells Connection to Contacts}

A good contact between CNTs and metal electrodes ensures a low resistance. Due to multiple shells in MWCNT, it could happen that some of the shells (i.e., inner shells) may not be connected to the contact. Disconnected shells do not contribute to the overall MWCNT conductance and can detriment its performance. Each CNT shell resistance $R_{i}$ is shown in Equations (3) and (4) [16]. $R_{Q}, N_{C}$ and $\lambda_{i}$ represent quantum resistance, number of conducting channels and mean free path (MFP) in the CNT shell $i$ respectively. $h / 2 e^{2} \sim 12.9 \mathrm{k} \Omega$ and $L$ is the CNT shell length. As shown in these equations, the scattering-induced shell resistance $R_{S i}$ is inversely proportional to the CNT shell diameter $D_{C N T i}$, which means the outer CNT shells have lower resistance than the inner shells. Thus, the MWCNT electrical performance is more susceptible when outer shells are disconnected rather than the inner shells. The calculation of $N_{C}$ at different shell diameters for both metallic and semiconducting CNTs is based on a Transmission Coefficients method [27], which is detailed in Part II.

$$
\begin{gathered}
R_{i}=R_{Q}+R_{S i} L=\frac{h}{2 e^{2} N_{C i}}+\frac{h}{2 e^{2} N_{C i}} \frac{L}{\lambda_{i}} \\
\lambda_{i} \approx 1000 D_{C N T i}
\end{gathered}
$$

In this work, we consider various scenarios of disconnected shells from contacts. On a $p$-shell MWCNT (i.e., the total number of shells is $p$ ), different shell numbers ranging from 1 to $p$ can be disconnected from the contacts. Depending on the number of shells disconnected and their location, the MWCNT performance will vary accordingly. For $n$ shells disconnected, the largest performance degradation of MWCNT corresponds if the outermost $n$ shells are disconnected while the least performance degradation corresponds if the innermost $n$ shells are disconnected.

\section{MWCNT PhySICAL COMPaCt Model}

\section{A. Original MWCNT Distributed Compact Model}

The original distributed MWCNT compact model of $p$-shell was proposed by [16]. Calculations of each parameter can be referred to [16] including intra-shell parameters such as scattering resistance $R_{S}$ and inter-shell parameters such as shell-to-shell capacitance $C_{S}$. Deriving the number of shells and diameter of each shell is shown in Equations (1) and (2).

\section{B. Enhanced MWCNT Distributed Compact Model}

Based on the original model, we develop an enhanced distributed MWCNT compact model considering defects and contact resistances as shown in Fig. 4. In the enhanced distributed model, the defect-induced resistance of each shell 


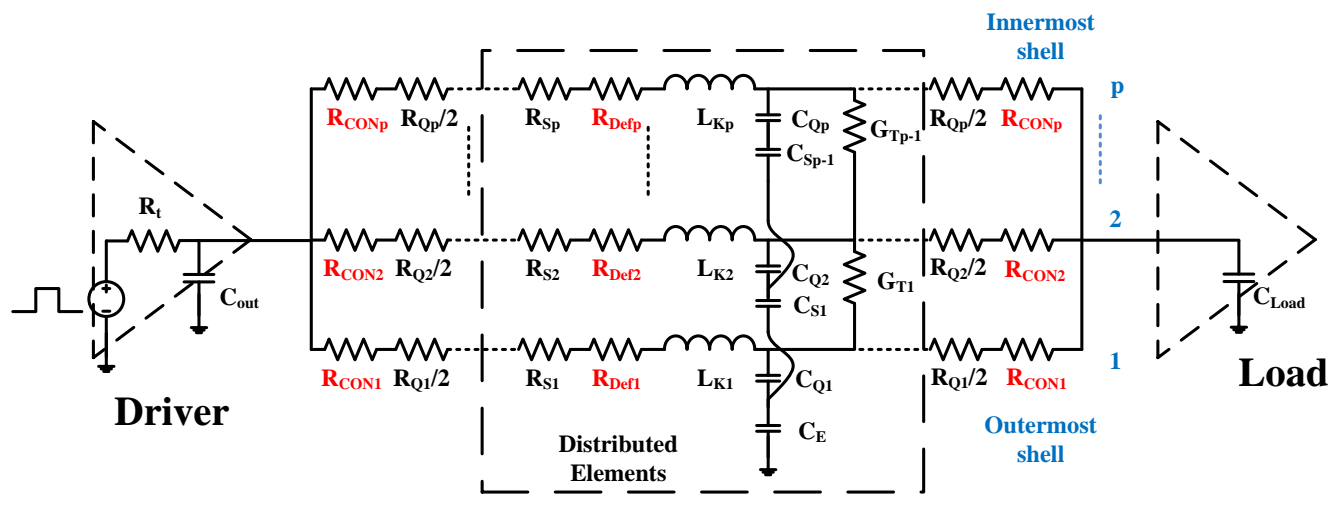

Fig. 4. Enhanced distributed $p$-shell MWCNT compact model proposed in this paper based on the original compact model by [16]. New parameters are contact resistance $R_{C O N}$ and defect-induced resistance $R_{D e f}$ in each shell. Except $R_{C O N}$ and lumped quantum resistance $R_{Q}$, each shell has intra-shell distributed parameters including $R_{D e f}$ and scattering resistance $R_{s}$, kinetic inductance $L_{K}$, and quantum capacitance $C_{Q}$. Inter-shell parameters include tunneling conductance $G_{T}$ and shell-to-shell capacitance $C_{S}$. Intra-shell parameters have $p$ components while inter-shell parameters have $p$-1 components. Only the outermost shell has electrostatic capacitance $C_{E}$ with the ground. $R_{t}$ and $C_{\text {out }}$ are the effective resistance and output capacitance of the driver, respectively, and $C_{l o a d}$ is the input capacitance of the load [16].

is distributed uniformly in the shell similar to the scattering CNT resistance. Different from the original model, the contact resistance of each shell is included which is diameter dependent. Furthermore, in the enhanced distributed compact model, the contribution of semiconducting CNT shells to the MWCNT overall performance is ignored. This is because the conductance of semiconducting CNT shells is several orders less than that of metallic CNT shells and thus negligible. Besides, the contact resistance between a semiconducting CNT and electrode is also several orders larger than the contact resistance between a metallic CNT and electrode.

\section{1) Defect Resistance:}

In [23], atomistic-level simulations showed that vacancy-type defects induced resistance $R_{\text {Def }_{i}}(\Omega)$ in metallic CNTs is on average proportional to the defect density $N_{D e f_{i}}(/ \mathrm{nm})$ and is inversely dependent on the shell diameter $D_{C N T_{i}}(\AA)$ as shown in Equation (5), where $L$ is the CNT shell length. It should be noted that $R_{D e f}$ is introduced to represent the impact of defects on the resistance of MWCNT and it could physically result from an increase in $R_{s}$ [23]. This defect-induced resistance is distributed uniformly along each MWCNT shell as shown in Fig. 4. Furthermore, we assume that the density of defect vacancies is not dependent on the diameter of each shell. As a result, $N_{D e f_{i}}$ is the same for each shell and the difference of $R_{D e f_{i}}$ between each shell results from their diameter difference.

$R_{D e f}\left(N_{D e f_{i}}, D_{C N T_{i}}\right)_{i}=2.67 \times 10^{5} \times N_{D e f_{i}} \times L \times D_{C N T_{i}}^{-1.27}$

2) Contact Resistance:

In this work, we perform atomistic-level simulation of a metallic armchair CNT with Density Function Theory (DFT) [28], [29]. The simulation temperature is fixed at $300 \mathrm{~K}$. We obtain that end-contact resistance of Pd-SWCNT is inversely dependent on the cross-sectional area of SWCNT as shown in Fig. 5, although different slopes are found and some fluctuation exists. We believe that the fluctuation is due to

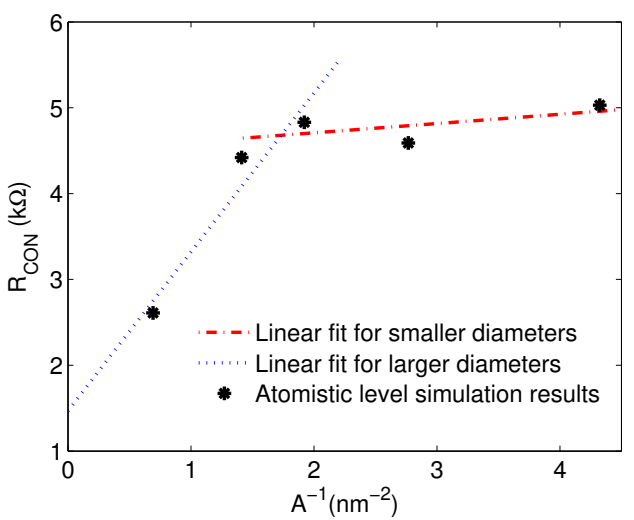

Fig. 5. Dependence of Pd-SWCNT end-contact resistance on cross-sectional area of SWCNT.

discretization of atom positions during DFT simulation, which leads to diameter dependent variation. For large diameters (such as diameter $D_{C N T}=0.8 \mathrm{~nm} \sim 1.3 \mathrm{~nm}$ ), a good linear fit is obtained by setting the reciprocal of CNT area $\left(A_{i}\left(\mathrm{~nm}^{2}\right)\right)$ as a variable while contact resistance $R_{C O N_{i}}$ changes with it. We obtain the coefficient of determination $R^{2}=0.9384$. As we study MWCNT with shell diameter $\geq 1 \mathrm{~nm}$, it is reasonable to use the linear fitting results for large diameters by extrapolation and derive the end-contact resistance as in Equation (6).

$$
R_{C O N_{i}}=1.8514 A_{i}^{-1}+1.4685 \quad(\mathrm{k} \Omega) \quad A_{i}=\frac{\pi D_{C N T_{i}}^{2}}{4}
$$

Although, the equation is based on the metallic armchair SWCNT simulation, the metallic zigzag CNT is assumed to have an identical contact resistance with Pd for the same shell diameter. Furthermore, the MWCNT contact resistance can be computed by considering each MWCNT shell as a SWCNT. Thus, according to this equation, the contact resistance of each shell can be calculated and introduced at the two contact-ends 


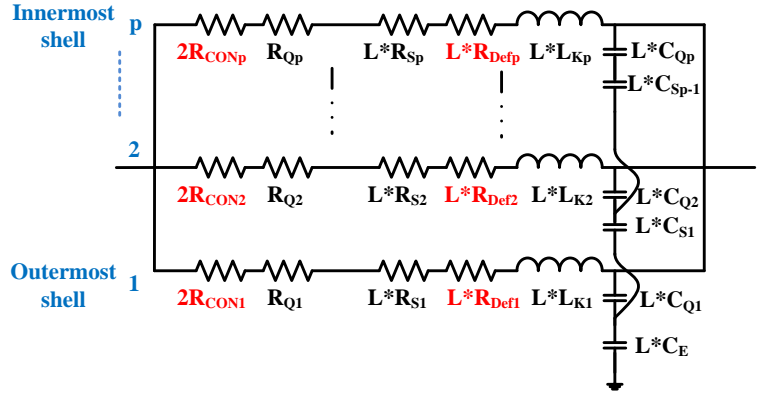

Fig. 6. Enhanced lumped $p$-shell MWCNT compact model proposed based on the enhanced distributed compact model in Fig. 4. Driver and load are not shown for clarity.

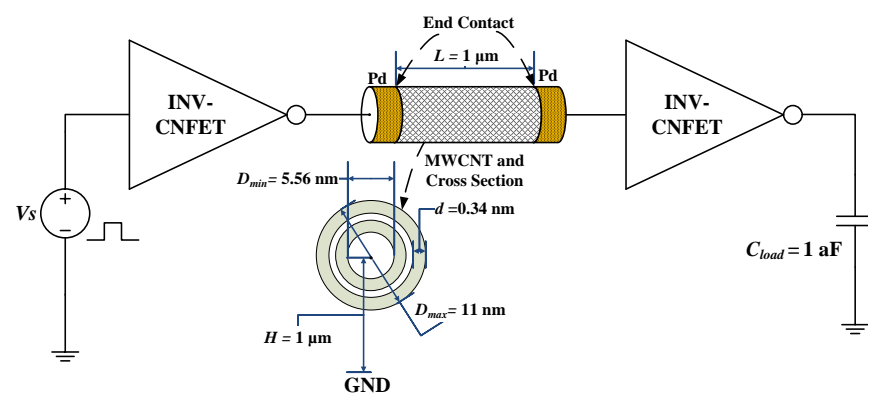

Fig. 7. Simulation setup schematic of MWCNT as an interconnect. MWCNT connects two inverters composed of CNTFET devices.

as shown in Fig. 4.

3) Enhanced Lumped Compact Model:

To simplify the enhanced distributed MWCNT compact model, we develop a respective lumped model as shown in Fig. 6. Compared with the distributed model in Fig. 4, the lumped model replaces the distributed parameters such as $R_{S}$ with lumped parameters by multiplying the distributed values with the MWCNT length. Tunneling parameters and shell-to-shell capacitance are eliminated as they are relatively negligible. Circuit level simulations using the simulation setup in Section IV show that for local interconnect application (such as MWCNT length $\leq 20 \mu \mathrm{m}$ ) the lumped model represents well the distributed model without any accuracy loss but significantly improves simulation efficiency. Thus, in our simulations, we use the lumped model.

\section{Simulation Setup and Results}

\section{A. Simulation setup}

The simulation setup schematic is shown in Fig. 7. The circuit benchmark consists of two inverters connected through either an ideal wire or a MWCNT interconnect. Inverter gates are built with carbon nanotube field effect transistors (CNTFETs), however other types of devices can also be used. Here, we use CNTFETs as devices with MWCNT interconnects to assess the performance benefits of a possible all carbonbased technology. We use CNTFET compact models proposed by [30]. The key parameters of both N-type and P-type CNTFET are $11.7 \mathrm{~nm}, 1 \mu \mathrm{m}, 10 \mathrm{~nm}$ for gate length, gate width and the distance between CNTs underneath the gate region, respectively. Other parameters are the default values recommended by [30]. The input stimulus is a square wave of $5 \mathrm{ps}$ rising and falling time. The period of the square wave is 100 ps. The supply voltage is $0.7 \mathrm{~V}$. The delay, power consumption, and product of power and delay (PDP) of the MWCNT interconnect are calculated by subtracting from the circuit respective values with an ideal connection from those with MWCNT interconnect. The outermost MWCNT diameter $D_{C N T \max }$ is $11 \mathrm{~nm}$ with length $L$ of $1 \mu \mathrm{m}$.

We perform MC simulations for diameter, defects, chirality and all-sources variations. The all-sources variation refers to the case that diameter, defects and chirality variations are simultaneously considered. The diameter, defect and chirality variations are randomized respectively as follows: $D_{C N T \max }$ (Gaussian distribution $\left.N\left(11 \mathrm{~nm}, 1.65^{2} \mathrm{~nm}^{2}\right)\right), R_{D e f}(\operatorname{LogN}$ distribution, $\ln \left(R_{D e f}\right) \sim N\left(\mu_{\log N},\left(\mu_{\log N} \times 0.0497\right)^{2}\right), \mu_{\log N}$ can be calculated based on the mean of $R_{D e f}$ in Equation (5)), and each CNT shell chirality (0-1 distribution, $1 / 3$ to be metallic). The all-sources variation has the above all parameters randomized simultaneously. For each run of MC simulation, 1000 samples are collected to obtain a reasonable confidence level for the respective simulation results. Defect density in the MWCNT is assumed to be 10 defects per $\mu \mathrm{m}(10 / \mu \mathrm{m})$ for each shell of MWCNT by default. The chirality variation that may generate all semiconducting MWCNT shells $(\sim 2.6 \%$ probability) can make MWCNT interconnect non-conducting and is thus not considered in the following simulation results.

\section{B. Diameter, Defects, Chirality and All-sources Variations}

Results of MWCNT resistance variations are shown in Fig. 8 (a)-(d). For each source of variation, distributions of delay, power, and PDP are found to be similar to their respective resistance variation and are thus not shown in detail. The similarity is due to the relatively negligible influence of MWCNT capacitance variation on these performance because MWCNT capacitance is $\sim 3$ orders smaller than the inverter parasitic capacitance (found by DC parasitic parameters extraction). Diameter variations can lead to either an increase or a decrease in the number of the MWCNT shells, which also makes MWCNT conductance change discretely as in Fig. $8(a)$. There are nine clusters in diameter variation which also corresponds to the total number of shells. Chirality variation is not continuous resulting from different combinations of metallic and semiconducting CNT shells in the MWCNT. Because metallic and semiconducting shells have significantly different conductance, chirality variation is significant as shown in Fig. $8(\mathrm{c})$. Defect and all-sources variations continuously change MWCNT conductance as shown in Fig. 8(b).

We find that $\operatorname{LogN}$ distributions can fit different sources variations. MWCNT resistance for diameter variation is not symmetrically varied around its mean value despite the Gaussian distribution of diameter variation as the change of resistance with decreasing diameters is larger than the change of resistance with increasing diameters. The chirality variation is also varied asymmetrically due to the asymmetrical variation of the portion of metallic CNT shells in MWCNT. Defect 


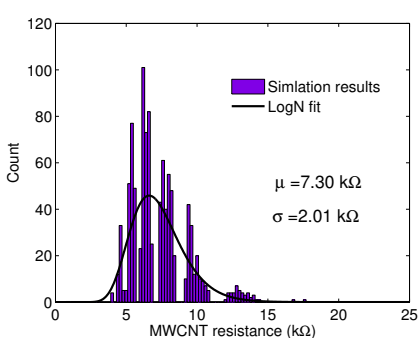

(a) Diameter

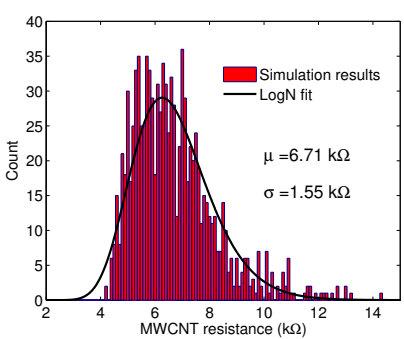

(b) Defects

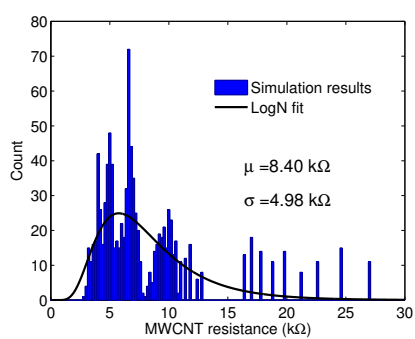

(c) Chirality

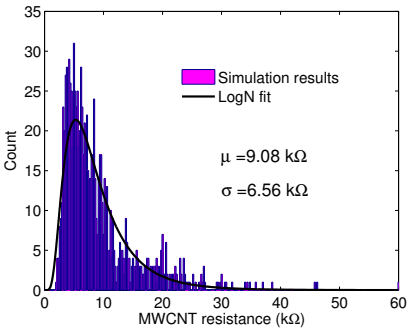

(d) All-sources

Fig. 8. (a)-(d) are distributions of resistance variation of the pristine MWCNT interconnect with input diameter, defects, chirality and all-sources variations respectively.

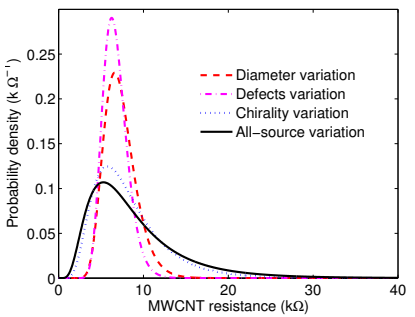

(a) Resistance

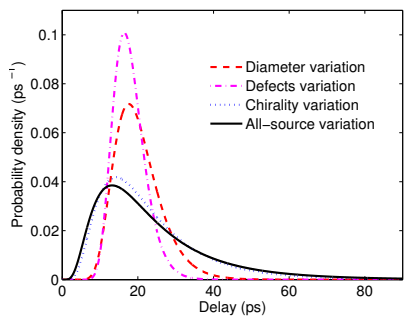

(b) Delay

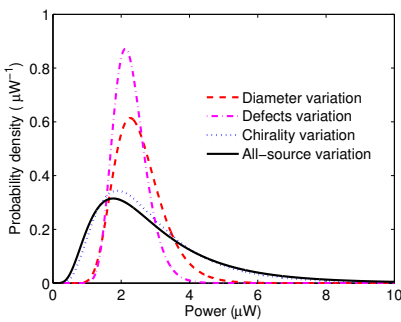

(c) Power

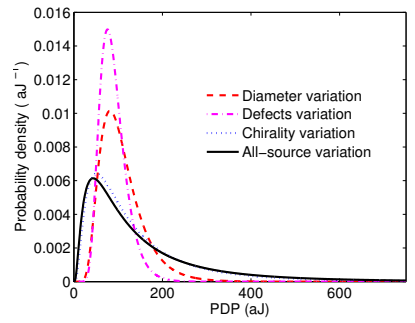

(d) PDP

Fig. 9. (a)-(d) are PDFs of resistance, delay, power and PDP of the pristine MWCNT interconnect respectively with input diameter, defects, chirality and all-sources variations.

variation complies also with a $\operatorname{LogN}$ distribution due to the input of $\log \mathrm{N}$ distributed defect-induced resistance. The all-sources variation is impacted by these three sources of variations and also shows $\log \mathrm{N}$ type distribution as in Fig. 8(d).

Additionally, we show mean $(\mu)$ and standard deviation $(\sigma)$ of MWCNT resistance for each source variation in Fig. 8(a)(d). We observe that the chirality is the most dominant source of variation, then diameter variation followed by defects as the least dominant source of variation. This can also be seen in Fig. 9(a)-(d) where the fitted probability density functions (PDFs) of each source of variation for resistance, delay, power and power-delay-product (PDP) are presented. The reason why chirality variation dominates is that chirality variation changes the portion of metallic CNT shells from $\sim 0$ to $\sim 1$, and hence significantly impacts resistance and performance of MWCNT. Diameter variation is also large because $D_{C N T \max }$ not only impacts $N_{C}$ but also influences the contact resistance and defect-induced resistance.

\section{Impact of Defect Density}

To investigate further the impact of defects, we vary defect densities ranging from $5 / \mu \mathrm{m}$ to $100 / \mu \mathrm{m}$. Please note that in previous subsection the defect density was set to $10 / \mu \mathrm{m}$. As shown in Fig. 10, $\mu$ and $\sigma$ of MWCNT resistance for each source of variation are increased linearly with defect density at slightly different rates. The increase in defect density leads to larger metallic CNT resistance and further the $\mu$ of MWCNT resistance. The increase in defect resistance $\sigma$ with defect density is as expected while the increase in diameter

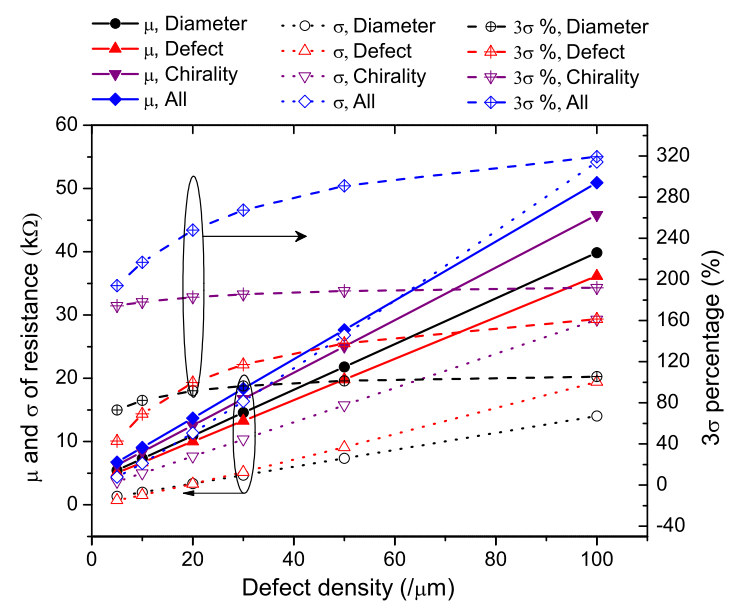

Fig. 10. Change of resistance while varying defect density on pristine MWCNT interconnects with diameter, defect, chirality and all-sources variations.

resistance $\sigma$ can be explained by Equation (5) where defectinduced resistance is dependent on both diameter and defect density. Additionally, the chirality variation $\sigma$ increases as each shell resistance increases with defect density.

The $3 \sigma$ percentage variation $(3 \sigma / \mu \times 100 \%)$ (or relative variation) [31] for each variation source also increases with defect density at different rates such as for small defect densities $(5 / \mu \mathrm{m}-50 / \mu \mathrm{m})$, as shown in Fig. 10. We observe that defect variation starts to surpass diameter variation for defect density of $\sim 15 / \mu \mathrm{m}$, but they are still less significant than the chirality variation. As defect density continues to increase, the 


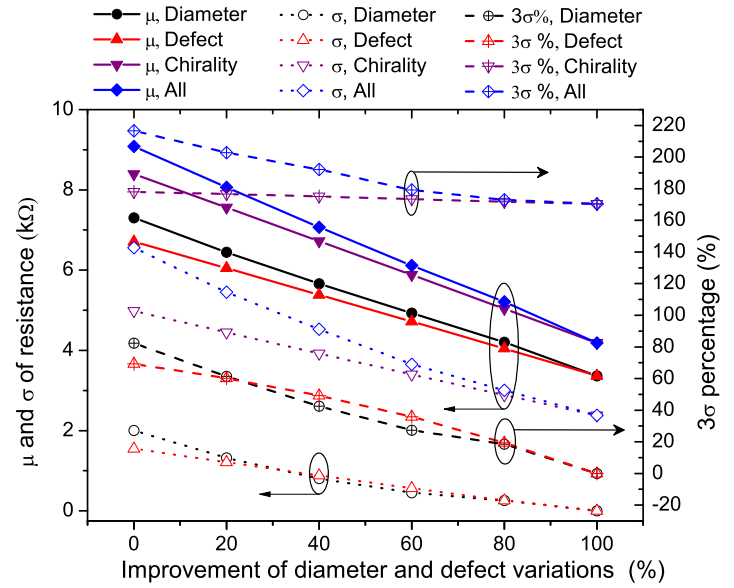

Fig. 11. Minimizing resistance variation of the pristine MWCNT interconnect by reducing the contribution of diameter and defect variations.

$3 \sigma$ percentage variation seems to saturate for different sources of variations. As a result, the chirality variation remains the most dominant source. Such observations suggest that chirality is the most dominant source of variations among all sources despite the large defect density.

\section{Improvement on MWCNT Variations}

Here, we investigate the scenario of an improved CNT growth method that can reduce MWCNT process variations. We consider improvements in defect and diameter variability whereas chirality variability remains unchanged as there is no trivial method to control chirality during growth. We investigate resistance variability as shown in Fig. 11 with different improvement levels of diameter and defect variations. The $\mathrm{x}$-axis indicates the percentage improvement in both defect density and diameter variability. We observe that resistance $\mu$, $\sigma$ and $3 \sigma$ percentage are reduced with the improvements on defect and diameter variability. However, the $3 \sigma$ percentage for chirality variation remains almost unchanged. This is because the resistance $\mu$ and $\sigma$ of chirality variation change at the same pace with the variability improvement. We notice, that when the percentage improvement reaches around $80 \%$, the chirality variation starts to be almost the only source of variation. We deduce that if process variations (such as diameter and defect) can be well controlled and reduced, then the chirality will be the only source of MWCNT variation that limits the improvement of MWCNT variability.

\section{E. MWCNT Shell to Contact Variations}

As described in Section II, we also investigate the connectivity between MWCNT shells and contacts. Good contacts between shell and metal electrodes are essential for achieving a low resistance contact. In this work, MWCNT interconnect has up to nine shells. We study the cases where the number of shells $n$ ranging from zero to nine can be disconnected from metal electrodes, which can lead to resistance variations and performance degradations. The case of all nine shells disconnected to contact would lead to conduction failure and

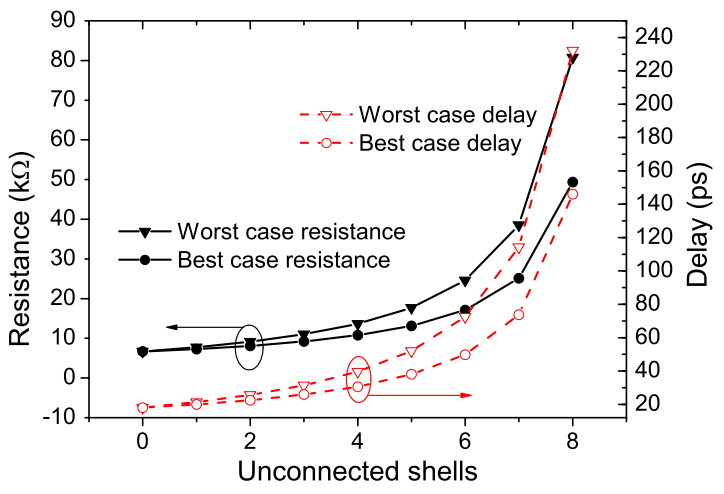

(a) Resistance and Delay

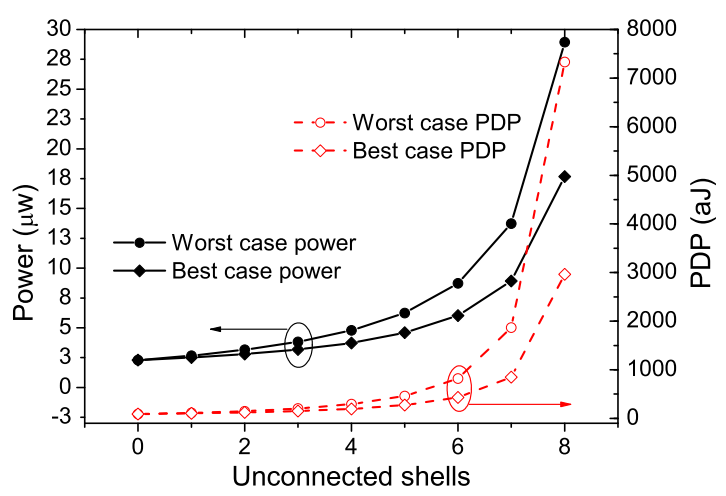

(b) Power and PDP

Fig. 12. Change of (a) resistance and delay, (b) power and PDP of MWCNT with disconnected CNT shells from contact. In this example, there are total nine shells in the MWCNT.

is not considered here. Simulation results in Fig. 12 show the MWCNT resistance, delay, power and PDP with respect to the number of disconnected shells. We observe significant (more than one order of magnitude) increases in resistance and performance degradations as the number of disconnected shells increases, especially for PDP. Depending on the positions of the disconnected shells (such as innermost or outermost), the performance degradation is also different. There are the best and the worst cases of performance degradation, which corresponds to all $n$ innermost shells disconnected and all $n$ outermost shells disconnected, respectively. We find that the difference between the two extreme cases gets larger with the number of disconnected shells.

\section{CONClusion}

In this paper, an enhanced compact model of MWCNT interconnect is presented where diameter dependent contact resistance and defect-induced resistance for each MWCNT shell are included. Based on the proposed enhanced compact model, MWCNT interconnect resistance and performance variations (including delay, power, and PDP) are analyzed including variations on diameter, defect densities, chirality, and shellto-contact connectivity. We identify that the diameter, defects, chirality, and all-sources variations all produce Lognormal resistance and performance distributions. We find that defect variation is increased with defect density at a larger rate than 
diameter variation and surpasses diameter variation at defect density of $\sim 15 / \mu \mathrm{m}$. The chirality variation is always the most dominant source of variation even at large defect densities. We also consider the case of significantly reduced MWCNT diameter and defect variations due to improving the quality of MWCNT growth, where the chirality remains as the only source of variation.

\section{ACKNOWLEDGMENT}

Authors would like to acknowledge H2020 CONNECT European project. This project has received funding from the European Union Horizon 2020 research and innovation program under grant agreement No 688612. (http://www.connecth2020.eu/).

\section{REFERENCES}

[1] S. Im, N. Srivastava, K. Banerjee, and K. E. Goodson, "Scaling analysis of multilevel interconnect temperatures for high-performance ics," IEEE Transactions on Electron Devices, vol. 52, no. 12, pp. 2710-2719, 2005. doi: 10.1109/ted.2005.859612

[2] W. Steinhögl, G. Schindler, G. Steinlesberger, M. Traving, and M. Engelhardt, "Comprehensive study of the resistivity of copper wires with lateral dimensions of $100 \mathrm{~nm}$ and smaller," Journal of Applied Physics, vol. 97, no. 2, p. 023706, 2005. doi: 10.1063/1.1834982

[3] A. Todri-Sanial, J. Dijon, and A. Maffucci, Carbon Nanotubes for Interconnects. Springer, 2017 ISBN: 978-3-319-29744-6. doi: 10.1007/9783-319-29746-0

[4] K. Liew, C. Wong, X. He, and M. Tan, "Thermal stability of single and multi-walled carbon nanotubes," Physical Review B, vol. 71, no. 7, p. 075424, 2005. doi: 10.1103/physrevb.71.075424

[5] B. Wei, R. Vajtai, and P. Ajayan, "Reliability and current carrying capacity of carbon nanotubes," Applied Physics Letters, vol. 79, no. 8, pp. 1172-1174, 2001. doi: 10.1063/1.1396632

[6] A. Todri-Sanial, R. Ramos, H. Okuno, J. Dijon, A. Dhavamani, M. Widlicenus, K. Lilienthal, B. Uhlig, T. Sadi, V. Georgiev et al., "A survey of carbon nanotube interconnects for energy efficient integrated circuits," IEEE Circuits and Systems Magazine, vol. 17, no. 2, pp. 47-62, 2017. doi: 10.1109/mcas.2017.2689538

[7] K. Banerjee and A. Mehrotra, "Global (interconnect) warming," IEEE Circuits and Devices Magazine, vol. 17, no. 5, pp. 16-32, 2001. doi:10.1109/101.960685

[8] A. K. Goel, High-Speed VLSI Interconnections. John Wiley \& Sons, Inc., aug 2007. doi:10.1002/9780470165973

[9] A. L. P. L. P. Petit, S. Roche, and J. Salvetat, Understanding Carbon Nanotubes, from Basics to Application. Lect. Notes Phys., édition Springer, 2006. doi: 10.1007/3-540-37586-4

[10] P. G. Collins, "Defects and disorder in carbon nanotubes," in $O x$ ford Handbook of Nanoscience and Technology Volume 2: Materials: Structures, Properties and Characterization Techniques. Oxford University Press Oxford, 2009, pp. 156-184. doi: 10.1093/oxfordhb/9780199533053.013.2

[11] M. Zhang and J. Li, "Carbon nanotube in different shapes," Materials today, vol. 12, no. 6, pp. 12-18, 2009. doi: 10.1016/s1369-7021(09)701762

[12] P. Poncharal, C. Berger, Y. Yi, Z. L. Wang, and W. A. de Heer, "Room temperature ballistic conduction in carbon nanotubes," The Journal of Physical Chemistry B, vol. 106, no. 47, pp. 12 104-12 118, 2002. doi: 10.1021/jp021271u

[13] A. Urbina, I. Echeverria, A. Pérez-Garrido, A. Díaz-Sánchez, and J. Abellán, "Quantum conductance steps in solutions of multiwalled carbon nanotubes," Physical review letters, vol. 90, no. 10, p. 106603 , 2003. doi: $10.1103 /$ physrevlett. 90.106603

[14] S. Esconjauregui, M. Fouquet, B. C. Bayer, C. Ducati, R. Smajda, S. Hofmann, and J. Robertson, "Growth of ultrahigh density vertically aligned carbon nanotube forests for interconnects," Acs Nano, vol. 4, no. 12 , pp. $7431-7436$, 2010. doi: $10.1021 / \mathrm{nn} 1025675$

[15] Q. Cao, S.-J. Han, J. Tersoff, A. D. Franklin, Y. Zhu, Z. Zhang, G. S. Tulevski, J. Tang, and W. Haensch, "End-bonded contacts for carbon nanotube transistors with low, size-independent resistance," Science, vol. 350 , no. 6256 , pp. 68-72, 2015. doi: 10.1126/science.aac8006
[16] H. Li, W.-Y. Yin, K. Banerjee, and J.-F. Mao, "Circuit modeling and performance analysis of multi-walled carbon nanotube interconnects," IEEE Transactions on electron devices, vol. 55, no. 6, pp. 1328-1337, 2008. doi: $10.1109 /$ ted.2008.922855

[17] A. Naeemi and J. D. Meindl, "Compact physical models for multiwall carbon-nanotube interconnects," IEEE Electron Device Letters, vol. 27, no. 5, pp. 338-340, 2006. doi: 10.1109/led.2006.873765

[18] H. Li, W. Lu, J. Li, X. Bai, and C. Gu, "Multichannel ballistic transport in multiwall carbon nanotubes," Physical review letters, vol. 95, no. 8, p. 086601, 2005. doi: 10.1103/physrevlett.95.086601

[19] J. Liang, R. Ramos, J. Dijon, H. Okuno, D. Kalita et al., "A physicsbased investigation of pt-salt doped carbon nanotubes for local interconnects," in Electron Devices Meeting (IEDM), 2017 IEEE International. IEEE, 2017, pp. 35-5. doi: 10.1109/iedm.2017.8268502

[20] P. Avouris, J. Appenzeller, R. Martel, and S. J. Wind, "Carbon nanotube electronics," Proceedings of the IEEE, vol. 91, no. 11, pp. 1772-1784, 2003. doi: $10.1109 /$ JPROC.2003.818338

[21] N. Chiodarelli, O. Richard, H. Bender, M. Heyns, S. De Gendt, G. Groeseneken, and P. M. Vereecken, "Correlation between number of walls and diameter in multiwall carbon nanotubes grown by chemical vapor deposition," Carbon, vol. 50, no. 5, pp. 1748-1752, 2012. doi: 10.1016/j.carbon.2011.12.020

[22] M. Bockrath, W. Liang, D. Bozovic, J. H. Hafner, C. M. Lieber, M. Tinkham, and H. Park, "Resonant electron scattering by defects in single-walled carbon nanotubes," Science, vol. 291, no. 5502, pp. 283285, 2001. doi: 10.1126/science.291.5502.283

[23] J. Lee, S. Berrada, J. Liang, T. Sadi, V. P. Georgiev, A. Todri-Sanial, D. Kalita, R. Ramos, H. Okuno, J. Dijon et al., "The impact of vacancy defects on cnt interconnects: From statistical atomistic study to circuit simulations," in Simulation of Semiconductor Processes and Devices (SISPAD), 2017 International Conference on. IEEE, 2017, pp. 157160. doi: $10.23919 /$ sispad.2017.8085288

[24] P. Holgate, "The lognormal characteristic function," Communications in Statistics-Theory and Methods, vol. 18, no. 12, pp. 4539-4548, 1989. doi: 10.1080/03610928908830173

[25] J. W. Wilder, L. C. Venema, A. G. Rinzler, R. E. Smalley, and C. Dekker, "Electronic structure of atomically resolved carbon nanotubes," Nature, vol. 391, no. 6662, p. 59, 1998. doi: 10.1038/34139

[26] Y. Massoud and A. Nieuwoudt, "Modeling and design challenges and solutions for carbon nanotube-based interconnect in future high performance integrated circuits," ACM Journal on Emerging Technologies in Computing Systems (JETC), vol. 2, no. 3, pp. 155-196, 2006. doi: $10.1145 / 1167943.1167944$

[27] H. H. B. Sørensen, P. C. Hansen, D. E. Petersen, S. Skelboe, and K. Stokbro, "Efficient wave-function matching approach for quantum transport calculations," Physical Review B, vol. 79, no. 20, p. 205322, 2009. doi: 10.1103/physrevb.79.205322

[28] M. Brandbyge, J.-L. Mozos, P. Ordejón, J. Taylor, and K. Stokbro, "Density-functional method for nonequilibrium electron transport," Physical Review B, vol. 65, no. 16, p. 165401, 2002. doi: 10.1103/physrevb.65.165401

[29] J. M. Soler, E. Artacho, J. D. Gale, A. García, J. Junquera, P. Ordejón, and D. Sánchez-Portal, "The siesta method for ab initio order-n materials simulation," Journal of Physics: Condensed Matter, vol. 14, no. 11, p. 2745, 2002. doi: 10.1088/0953-8984/14/11/302

[30] C.-S. Lee, E. Pop, A. D. Franklin, W. Haensch, and H.-S. Wong, "A compact virtual-source model for carbon nanotube fets in the sub-10-nm regimepart i: Intrinsic elements," IEEE transactions on electron devices, vol. 62 , no. 9, pp. 3061-3069, 2015. doi: 10.1109/ted.2015.2457453

[31] A. Nieuwoudt and Y. Massoud, "On the impact of process variations for carbon nanotube bundles for vlsi interconnect," IEEE Transactions on Electron Devices, vol. 54, no. 3, pp. 446-455, 2007. doi: 10.1109/ted.2006.890364 